\title{
GLOBAL EXISTENCE AND ASYMPTOTIC BEHAVIOUR OF SOLUTIONS FOR A CLASS OF FOURTH ORDER STRONGLY DAMPED NONLINEAR WAVE EQUATIONS
}

BY

XU RUNZHANG (College of Science, Harbin Engineering University, 150001, People's Republic of China )

AND

YANG YANBING (College of Science, Harbin Engineering University, 150001, People's Republic of China )

Abstract. In this paper we study the initial boundary value problem for a class of fourth order strongly damped nonlinear wave equations $u_{t t}-\Delta u+\Delta^{2} u-\alpha \Delta u_{t}=f(u)$. By introducing a family of potential wells we prove the existence of global weak solutions and global strong solutions under some weak growth conditions on $f(u)$. Furthermore we give the asymptotic behaviour of solutions.

1. Introduction. In this paper we study the initial boundary value problem (IBVP) of fourth order strongly damped nonlinear wave equations

$$
\begin{aligned}
& u_{t t}-\Delta u+\Delta^{2} u-\alpha \Delta u_{t}=f(u), x \in \Omega, t>0, \\
& u(x, 0)=u_{0}, u_{t}(x, 0)=u_{1}, x \in \Omega, \\
& u(x, t)=\Delta u(x, t)=0, x \in \partial \Omega, t \geq 0,
\end{aligned}
$$

where $\alpha$ is a positive constant and $\Omega \subset \mathbb{R}^{n}$ is a bounded domain with a smooth boundary $\partial \Omega$.

The strongly damped nonlinear wave equation

$$
u_{t t}-\Delta u-\alpha \Delta u_{t}=f(u)
$$

was suggested from the motion of a viscous body. In 1980, Webb [23 first studied the IBVP of (1.4) with the initial boundary value condition (1.2) and

$$
u(x, t)=0, x \in \partial \Omega, t \geq 0,
$$

Received May 27, 2011.

2010 Mathematics Subject Classification. Primary 35L25, 35A01, 35L30.

Key words and phrases. Fourth order nonlinear wave equations, strong damping, global existence, asymptotic behaviour, potential well.

E-mail address: xurunzh@yahoo.com.cn 
for $n \leq 3$. He obtained the global existence and asymptotic behaviour of the strong solution. The authors in [1] further considered the problem (1.4), (1.2) and (1.5). They strengthened the hypotheses and assumptions in 23] by proving the local and global existence of the solution and the exponential decay. Subsequently in [14] the same problem was studied again and the results in [23] were improved. In [13] the global existence of the strong solution to problem (1.4), (1.2), (1.5) was given for $n \geq 4$, which generalized the results obtained in 23 and [14. Then in [24] the authors further showed asymptotic behaviour of the global strong solution of problem (1.4), (1.2), (1.5). Here we would like to mention that the above results in [14, 13, 23, 24, are based on the assumption that (1.4) has a positive definite energy. In [15], the authors made the first try to consider the problem with nonpositive energy. They showed the global existence of both weak and strong solutions to problem (1.4), (1.2), (1.5) by the potential well method. This technique was taken to treat more complex problems such as (1.4). Newly, Pata and Squassina [19] investigated the following 3-D wave equation:

$$
u_{t t}-\Delta u-\alpha \Delta u_{t}+\phi(u)=f(u)
$$

with (1.4) and (1.5). They carried out a detailed analysis of the asymptotic behaviour of the solution independent of the damping coefficient. Subsequently Filippo and Squassina [7] considered (1.4) with weak damping $u_{t}$. They showed the global existence and the polynomial decay property of solutions provided that the initial data are in the potential well and $E(0)<d$. Recently Gerbi and Said-Houari [8] considered the same problem. They proved that the solution decays exponentially by introducing an appropriate Lyapunov function; hence the results in [7] were improved. Levine and Serrin [10] considered the global nonexistence of solutions for equations with weak damping

$$
u_{t t}-\Delta u+u_{t}=f(u) .
$$

They proved that the solutions with negative initial energy blow up in finite time. Then the case in [10] was extended to the abstract version by Levine, Pucci and Serrin [9]. Subsequently, Pucci and Serrin [21] improved the results from $E(0)<0$ to $E(0)<d$. Vitillaro 22] showed that the case also holds when $E(0)=d$. For the strongly damped equation, Ono [18] obtained that the solution of (1.4) blows up in finite time if $E(0)<0$. Later Ohta [17] improved this result provided that the initial data are out of the potential well and $E(0)<d$. Moreover there are many results about the global existence and asymptotic behaviour of solutions for the strongly damped wave equations; we refer the reader to [20, 2, 3, 4] and the references therein.

Less well known is the fact that there are a few results about the nonlinear fourth order equation (1.1) with $\alpha=0$. In [6], Esquivel studied the IBVP of equation

$$
u_{t t}+\Delta^{2} u-\alpha \Delta u+\sum_{i=1}^{n} \frac{\partial}{\partial x_{i}} \sigma_{i}\left(u_{x_{i}}\right)=f(u)
$$

for $\sigma_{i}(s)= \pm \beta|s|^{m-2} s$ and $f(u)=\mu|s|^{r-2} s$. By using the potential well method, the author obtained a sharp condition for global existence and nonexistence of the solution. Then in [16] these results were extended to more general nonlinear terms $\sigma_{i}(s)$ and $f(s)$ by employing a family of potential wells. Subsequently Chen and Ren [5] studied the 
existence, nonexistence, uniqueness and energy decay estimate of the solution for the IBVP of (1.1) with the nonlinear weak damping $\left|u_{t}\right|^{r} u_{t}$. By the multiplier method, they estimated the energy decay rate of the global solution. Most recently, in [11, by a potential well argument also, Lin, Wu and Lai considered the problem (1.1)-(1.3) and proved the existence of a global weak and strong solution under some assumptions on $f(u)$ and initial data. This is the first try to treat this problem, and they left some other interesting problems open. Next, in order to explain the motivation of this paper, we would like to show some of the results obtained in [11]. First we list some definitions in [1] as follows:

$$
\begin{aligned}
& \left(\mathrm{A}_{1}\right) \quad|f(u)| \leq a|u|^{p}, 1<p<\infty \text { if } n=1,2 ; 1<p \leq \frac{n-2}{n+2} \text { if } n \geq 3 . \\
& J(u)=\frac{1}{2}\|\nabla u\|^{2}+\frac{1}{2}\|\Delta u\|^{2}-\frac{a}{p+1}\|u\|_{p+1}^{p+1}, \\
& I(u)=\|\nabla u\|^{2}+\|\Delta u\|^{2}-a\|u\|_{p+1}^{p+1}, \\
& E(t)=\frac{1}{2}\left\|u_{t}\right\|^{2}+\frac{1}{2}\|\nabla u\|^{2}+\frac{1}{2}\|\Delta u\|^{2}-\int_{\Omega} F(u) \mathrm{d} x, F(u)=\int_{0}^{u} f(s) \mathrm{d} s, \\
& W=\left\{u \in H^{2}(\Omega) \cap H_{0}^{1}(\Omega) \mid I(u)>0, J(u)<d\right\} \cup\{0\}, \\
& V=\left\{u \in H^{2}(\Omega) \cap H_{0}^{1}(\Omega) \mid I(u)<0, J(u)<d\right\},
\end{aligned}
$$

where

$$
d=\inf _{\substack{u \in H^{2}(\Omega) \cap H_{0}^{1}(\Omega) \\ u \neq 0}} \sup _{\lambda \geq 0} J(\lambda u)
$$

$\left(\mathrm{A}_{2}\right) \quad\left|f^{\prime}(u)\right| \leq A|u|^{p_{1}}, \frac{1}{2}<p_{1}<\infty$ if $n=1,2 ; \frac{2}{n}<p_{1} \leq \frac{4}{n-2}$ if $n \geq 3$,

$$
B_{R}=\left\{u \in H^{2}(\Omega) \cap H_{0}^{1}(\Omega) \mid\left(\|\nabla u\|^{2}+\|\Delta u\|^{2}\right)^{\frac{1}{2}}<R\right\},
$$

where

$$
R=\left(a C_{*}^{p+1}\right)^{\frac{1}{p-1}}, \quad C_{*}=\sup _{\substack{u \in H^{2}(\Omega) \cap H_{0}^{1}(\Omega) \\ u \neq 0}} \frac{\|u\|_{p+1}}{\left(\|\nabla u\|^{2}+\|\Delta u\|^{2}\right)^{\frac{1}{2}}} .
$$

The main results of [1] are the following two theorems.

Theorem 1.1 ([1] $)$. Let $f \in C, f(u) u \geq 0$ and satisfy $\left(\mathrm{A}_{1}\right), u_{0} \in H^{2}(\Omega) \cap H_{0}^{1}(\Omega)$, $u_{1} \in L^{2}(\Omega)$. Assume that $E(0)<d$ and $u_{0} \in W$. Then problem (1.1)-(1.3) admits a global weak solution $u \in L^{\infty}\left(0, \infty ; H^{2}(\Omega) \cap H_{0}^{1}(\Omega)\right)$ with $u_{t} \in L^{\infty}\left(0, \infty ; L^{2}(\Omega)\right)$ and $u \in W$ for $0 \leq t<\infty$.

Theorem 1.2 ([11]). Let $f \in C^{1}, f(u) u \geq 0$ and satisfy $\left(\mathrm{A}_{2}\right), u_{0} \in H^{4}(\Omega) \cap H_{0}^{1}(\Omega)$, $u_{1} \in H^{2}(\Omega) \cap H_{0}^{1}(\Omega)$. Assume that $E(0)<d$ and $u_{0} \in B_{R}$. Then problem (1.1)(1.3) admits a unique global strong solution $u \in L^{\infty}\left(0, T ; H^{4}(\Omega) \cap H_{0}^{1}(\Omega)\right)$ with $u_{t} \in$ $L^{\infty}\left(0, T ; H^{2}(\Omega) \cap H_{0}^{1}(\Omega)\right), \forall T>0$ and $u \in B_{R}$ for $0 \leq t<\infty$.

The above theorems conclude the global-in-time existence of a solution. Further, for the damped nonlinear hyperbolic equation, we usually desire to know how the solutions behave as the time $t$ approaches infinity, the so-called asymptotic behaviour. To be more clear, we expect the solutions to decay to zero as $t \rightarrow \infty$. So this is one of the main tasks of the present paper. On the other hand, we would also like to focus on weakening 
the assumptions $\left(A_{1}\right)$ and $\left(A_{2}\right)$. Before doing these, we need to vary the definition of the strong solution, because by paying attention to the definition of the strong solution in 11 we find that from $u \in H^{4}(\Omega) \cap H_{0}^{1}(\Omega)$ one can only see $\left.u\right|_{\partial \Omega}=0$ rather than $\left.\Delta u\right|_{\partial \Omega}=0$. Hence the strong solution defined in this way cannot satisfy the boundary condition $\left.\Delta u\right|_{\partial \Omega}=0$. In order to solve this problem we introduce the space $H_{4}$ defined as below.

$H_{4}:=$ closed linear extension of $\left\{w_{j}(x)\right\}_{j=1}^{\infty}$ in $H^{4}(\Omega)$, where $\left\{w_{j}(x)\right\}_{j=1}^{\infty}$ is the eigenfunction system of problem $-\Delta w=\lambda w$ in $\Omega,\left.w\right|_{\partial \Omega}=0$.

Note that for any $w_{j}(x)$ we have $\left.\Delta w_{j}\right|_{\partial \Omega}=-\left.\lambda_{j} w_{j}\right|_{\partial \Omega}=0$. Hence for any $u \in H_{4}$ we have $u \in H^{4}(\Omega) \cap H_{0}^{1}(\Omega)$ and $\Delta u \in H^{2}(\Omega) \cap H_{0}^{1}(\Omega)$, which implies that $\left.u\right|_{\partial \Omega}=\left.\Delta u\right|_{\partial \Omega}=0$. Thus we can equivalently define $H_{4}$ by

$$
H_{4}:=\left\{u \mid \quad u \in H^{4}(\Omega) \cap H_{0}^{1}(\Omega), \Delta u \in H^{2}(\Omega) \cap H_{0}^{1}(\Omega) \text { with }\left.u\right|_{\partial \Omega}=\left.\Delta u\right|_{\partial \Omega}=0\right\} .
$$

In addition we denote $H^{2}(\Omega) \cap H_{0}^{1}(\Omega)$ by $H$. Then we give a new definition of a strong solution.

Definition 1.3. Function $u=u(x, t)$ is called a strong solution of problem (1.1) (1.3) on $\Omega \times[0, T)$ if $u \in L^{\infty}\left(0, T ; H_{4}\right)$ with $u_{t} \in L^{\infty}(0, T ; H), u_{t t} \in L^{\infty}\left(0, T ; L^{2}(\Omega)\right)$ satisfying

$$
\int_{0}^{T}\left(u_{t t}-\Delta u+\Delta^{2} u-\alpha \Delta u_{t}-f(u), \varphi\right) \mathrm{d} t=0, \forall \varphi \in C\left([0, T), L^{2}(\Omega)\right)
$$

and $u(x, 0)=u_{0}$ in $H_{4}, u_{t}(x, 0)=u_{1}$ in $H$.

Now we are ready to study the problem (1.1)-(1.3), where $\alpha$ is a positive constant, $\Omega \subset \mathbb{R}^{n}$ is a bounded domain with a smooth boundary $\partial \Omega$, and $f(u)$ satisfies

$$
\left(\mathrm{H}_{1}\right) \begin{cases}(i) \quad & f \in C^{1} \text { and } u\left(u f^{\prime}(u)-f(u)\right) \geq 0, \\ & \text { where the equality holds only for } u=0 ; \\ (i i) \quad & |f(u)| \leq A|u|^{q}, 1<q<\infty \text { for } 1 \leq n \leq 4 ; 1<q<\frac{n+4}{n-4} \text { for } n \geq 5 ; \\ (\text { iii }) \quad & \text { there exists a } p>1 \text { such that } \\ & (p+1) F(u) \leq u f(u), \forall u \in \mathbb{R}, F(u)=\int_{0}^{u} f(s) \mathrm{d} s,\end{cases}
$$

or

$$
\left(\mathrm{H}_{2}\right) \begin{cases}(i) \quad & f \in C^{1} \text { and } u\left(u f^{\prime}(u)-f(u)\right) \geq 0 \\ & \text { where the equality holds only for } u=0 ; \\ & f(0)=0,\left|f^{\prime}(u)\right| \leq a|u|^{q_{1}} \\ & 0<q_{1}<\infty \text { for } 1 \leq n \leq 4 ; 0<q_{1}<\frac{n}{n-4} \text { for } n=5,6 ; \\ & 0<q_{1}<\frac{6}{n-4} \text { for } n \geq 7 ; \\ (\text { iii }) \quad & \text { there exists a } p>1 \text { such that } \\ & (p+1) F(u) \leq u f(u), \forall u \in \mathbb{R}, F(u)=\int_{0}^{u} f(s) \mathrm{d} s .\end{cases}
$$

We organize this paper as follows: in Section 2, we introduce a family of potential wells and discuss the invariant set. In Section 3, we give the existence of global weak solutions under the assumption $\left(\mathrm{H}_{1}\right)$, and we also prove the existence of global strong solutions under the assumption $\left(\mathrm{H}_{2}\right)$. In Section 4, we obtain the asymptotic behaviour of global weak solutions for problem (1.1)-(1.3). For a clear presentation, we use the following 
denotation throughout the paper: $\|\cdot\|_{p}=\|\cdot\|_{L^{p}(\Omega)},\|\cdot\|=\|\cdot\|_{2},\|u\|_{H}^{2}=\|\nabla u\|^{2}+\|\Delta u\|^{2}$, $\|u\|_{H^{4}}^{2}=\left\|\Delta^{2} u\right\|^{2}+\left\|\nabla^{3} u\right\|^{2}, \nabla^{3}=\nabla(\Delta u),(u, v)=\int_{\Omega} u v \mathrm{~d} x$.

2. Preliminary lemmas. In this section we give some definitions and lemmas related to the potential wells. First for problem (1.1)-(1.3) we define

$$
\begin{aligned}
& J(u)=\frac{1}{2}\|\nabla u\|^{2}+\frac{1}{2}\|\Delta u\|^{2}-\int_{\Omega} F(u) \mathrm{d} x=\frac{1}{2}\|u\|_{H}^{2}-\int_{\Omega} F(u) \mathrm{d} x, \\
& I(u)=\|\nabla u\|^{2}+\|\Delta u\|^{2}-\int_{\Omega} u f(u) \mathrm{d} x=\|u\|_{H}^{2}-\int_{\Omega} u f(u) \mathrm{d} x, \\
& I_{\delta}(u)=\delta\left(\|\nabla u\|^{2}+\|\Delta u\|^{2}\right)-\int_{\Omega} u f(u) \mathrm{d} x=\delta\|u\|_{H}^{2}-\int_{\Omega} u f(u) \mathrm{d} x, \delta>0, \\
& d=\inf _{u \in \mathcal{N}} J(u), \mathcal{N}=\{u \in H \mid I(u)=0, u \neq 0\}, \\
& d(\delta)=\inf _{u \in \mathcal{N}_{\delta}} J(u), \mathcal{N}_{\delta}=\left\{u \in H \mid I_{\delta}(u)=0, u \neq 0\right\}, \\
& E(t)=\frac{1}{2}\left\|u_{t}\right\|^{2}+\frac{1}{2}\|\nabla u\|^{2}+\frac{1}{2}\|\Delta u\|^{2}-\int_{\Omega} F(u) \mathrm{d} x=\frac{1}{2}\left\|u_{t}\right\|^{2}+J(u) .
\end{aligned}
$$

Clearly if $f(u)$ satisfies $\left(\mathrm{H}_{1}\right)$ (or $\left(\mathrm{H}_{2}\right)$ ), then all of the above definitions are well defined for $u \in H^{2}(\Omega) \cap H_{0}^{1}(\Omega)$ (or $\left.u \in H^{4}(\Omega) \cap H_{0}^{1}(\Omega)\right)$.

Lemma 2.1. Let $f(u)$ satisfy $\left(\mathrm{H}_{1}\right)$ (or $\left.\left(\mathrm{H}_{2}\right)\right), u \in H$ (or $\left.u \in H_{4}\right)$ and $g(u)=\frac{f(u)}{u}, u \neq 0$. Then

(i) $\lim _{u \rightarrow 0} g(u)=0$;

(ii) $g(u)$ is strictly increasing on $(0, \infty)$, strictly decreasing on $(-\infty, 0)$;

(iii) $f(u) u>0, \forall u \neq 0$;

(iv) $f(u)$ is strictly increasing on $(-\infty, \infty)$;

(v) $0 \leq F(u) \leq A_{1}|u|^{q+1}$ or $A_{2}|u|^{q_{1}+2}, \forall u \in \mathbb{R}$.

Proof. (i) The conclusion follows directly from (ii) in $\left(\mathrm{H}_{1}\right)$ or $\left(\mathrm{H}_{2}\right)$.

(ii) From (i) in $\left(\mathrm{H}_{1}\right)$ or $\left(\mathrm{H}_{2}\right)$ we get

$$
g^{\prime}(u)=\frac{u f^{\prime}(u)-f(u)}{u^{2}}\left\{\begin{array}{l}
>0 \text { if } u>0 \\
<0 \text { if } u<0
\end{array}\right.
$$

(iii) From (i) and (ii) of this lemma we see that $g(u)>0$ for any $u \neq 0$, which gives $f(u) u>0$ for any $u \neq 0$.

(iv) From (i) in $\left(\mathrm{H}_{1}\right)$ or $\left(\mathrm{H}_{2}\right)$, and (iii) of this lemma we get

$$
f^{\prime}(u) \geq \frac{f(u)}{u}>0, \quad \forall u \neq 0 .
$$

(v) From $f(0)=0$ and (ii) of this lemma we have $F(u) \geq 0$, and $F(u) \leq A_{1}|u|^{q+1}$ or $A_{2}|u|^{q_{1}+2}$ follows from (ii) in $\left(\mathrm{H}_{1}\right)$ or $\left(\mathrm{H}_{2}\right)$.

Next we will give some lemmas to assist us to state and prove the main theorems. We shall not prove them, because we can get them by letting $\sigma_{i}(s) \equiv 0(1 \leq i \leq n)$ and $\alpha=1$ in Lemma 2.1-Lemma 2.9 in [16]. 
Lemma 2.2. Let $f(u)$ satisfy $\left(\mathrm{H}_{1}\right), u \in H$, or $f(u)$ satisfy $\left(\mathrm{H}_{2}\right), u \in H_{4}$. Then

$$
F(u) \geq B|u|^{p+1}, \forall|u| \geq 1 \text { and some } B>0,
$$

and

$$
u f(u) \geq(p+1) B|u|^{p+1}, \forall|u| \geq 1 .
$$

Lemma 2.3. Let $f(u)$ satisfy $\left(\mathrm{H}_{1}\right), u \in H$, or $f(u)$ satisfy $\left(\mathrm{H}_{2}\right), u \in H_{4}, \varphi(\lambda)=$ $\frac{1}{\lambda} \int_{\Omega} u f(\lambda u) \mathrm{d} \lambda, \lambda>0$. Then

(i) $\lim _{\lambda \rightarrow 0} \varphi(\lambda)=0, \lim _{\lambda \rightarrow+\infty} \varphi(\lambda)=+\infty$;

(ii) $\varphi(\lambda)$ is strictly increasing on $(-\infty,+\infty)$.

Lemma 2.4. Let $f(u)$ satisfy $\left(\mathrm{H}_{1}\right), u \in H, u \neq 0$, or $f(u)$ satisfy $\left(\mathrm{H}_{2}\right), u \in H_{4}, u \neq 0$. Then

(i) $\lim _{\lambda \rightarrow 0} J(\lambda u)=0, \lim _{\lambda \rightarrow+\infty} J(\lambda u)=-\infty$;

(ii) there exists a unique $\lambda^{*}=\lambda^{*}(u)$ such that

$$
\left.\frac{\mathrm{d}}{\mathrm{d} \lambda} J(\lambda u)\right|_{\lambda=\lambda^{*}}=0
$$

(iii) $J(\lambda u)$ is increasing on $0<\lambda \leq \lambda^{*}$, decreasing on $\lambda^{*} \leq \lambda<\infty$ and takes the maximum at $\lambda=\lambda^{*}$

(iv) $I(\lambda u)>0$ for $0<\lambda<\lambda^{*} ; I(\lambda u)<0$ for $\lambda^{*}<\lambda<\infty$ and $I\left(\lambda^{*} u\right)=0$.

Lemma 2.5. Let $f(u)$ satisfy $\left(\mathrm{H}_{1}\right), u \in H$, or $f(u)$ satisfy $\left(\mathrm{H}_{2}\right), u \in H_{4}$. Then:

(i) If $0<\|u\|_{H}<r(\delta)$, then $I_{\delta}(u)>0$.

(ii) If $I_{\delta}(u)<0$, then $\|u\|_{H}>r(\delta)$.

(iii) If $I_{\delta}(u)=0, u \neq 0$, then $\|u\|_{h} \geq r(\delta)$,

$$
\begin{gathered}
r(\delta)=\left(\frac{\delta}{A C_{*}^{q+1}}\right)^{\frac{1}{q-1}}, C_{*}=\sup _{\substack{u \in H \\
u \neq 0}} \frac{\|u\|_{q+1}}{\|u\|_{H}} \text { if } f(u) \text { satisfies }\left(H_{1}\right) ; \\
r(\delta)=\left(\frac{\left(q_{1}+1\right) \delta}{a C_{*}^{q_{1}+2}}\right)^{\frac{1}{q_{1}}}, C_{*}=\sup _{\substack{u \in H \\
u \neq 0}} \frac{\|u\|_{q_{1}+2}}{\|u\|_{H}} \text { if } f(u) \text { satisfies }\left(H_{2}\right) .
\end{gathered}
$$

Lemma 2.6. Let $f(u)$ satisfy $\left(\mathrm{H}_{1}\right), u \in H$, or $f(u)$ satisfy $\left(\mathrm{H}_{2}\right), u \in H_{4}$. Then

(i) $d(\delta) \geq a(\delta) r^{2}(\delta)$ for $a(\delta)=\frac{1}{2}-\frac{\delta}{p+1}, 0<\delta<\frac{p+1}{2}$;

(ii) $\lim _{\delta \rightarrow+\infty} d(\delta)=0$ and there exists a $0<\delta_{0} \leq \frac{p+1}{2}$ such that $d\left(\delta_{0}\right)=0$ and $d(\delta)>0$ for $0<\delta<\delta_{0}$

(iii) $d(\delta)$ is increasing on $0<\delta \leq 1$, decreasing on $1 \leq \delta<\delta_{0}$ and takes the maximum at $\delta=1$.

Now for problem (1.1) - (1.3) we define the following sets; later we will state the invariance of them.

$$
\begin{aligned}
& W=\{u \in H \mid I(u)>0, J(u)<d\} \cup\{0\}, \\
& W_{\delta}=\left\{u \in H \mid I_{\delta}(u)>0, J(u)<d(\delta)\right\} \cup\{0\}, 0<\delta<\delta_{0}, \\
& V=\{u \in H \mid I(u)<0, J(u)<d\}, \\
& V_{\delta}=\left\{u \in H \mid I_{\delta}(u)<0, J(u)<d(\delta)\right\}, 0<\delta<\delta_{0} .
\end{aligned}
$$


In addition we define

$$
\begin{aligned}
& W^{\prime}=\{u \in H \mid I(u)>0\} \cup\{0\}, \\
& W_{\delta}^{\prime}=\left\{u \in H \mid I_{\delta}(u)>0\right\} \cup\{0\}, 0<\delta<\delta_{0}, \\
& V^{\prime}=\{u \in H \mid I(u)<0\}, \\
& V_{\delta}^{\prime}=\left\{u \in H \mid I_{\delta}(u)<0\right\}, 0<\delta<\delta_{0} .
\end{aligned}
$$

The above definitions are valid only for $f(u)$ satisfying $\left(\mathrm{H}_{1}\right)$. For $f(u)$ satisfying $\left(\mathrm{H}_{2}\right)$, we can replace $u \in H$ by $u \in H_{4}$ in all of the above definitions. Now we shall give two theorems to state the invariance of the above manifolds.

Theorem 2.7. Let $f(u)$ satisfy $\left(\mathrm{H}_{1}\right), u_{0} \in H, u_{1} \in L^{2}(\Omega)$. Assume that $0<E(0)<d$, and let $\left(\delta_{1}, \delta_{2}\right)$ be the maximal interval such that $d(\delta)>E(0)$ for $\delta \in\left(\delta_{1}, \delta_{2}\right)$. Then:

(i) All weak solutions of problem (1.1)-(1.3) belong to $W_{\delta}$ for $\delta \in\left(\delta_{1}, \delta_{2}\right)$ provided that $u_{0} \in W^{\prime}$.

(ii) All weak solutions of problem (1.1) - (1.3) belong to $V_{\delta}$ for $\delta \in\left(\delta_{1}, \delta_{2}\right)$ provided that $u_{0} \in V^{\prime}$.

Theorem 2.8. Let $f(u)$ satisfy $\left(\mathrm{H}_{2}\right), u_{0} \in H_{4}, u_{1} \in H$. Assume that $0<E(0)<d$, and let $\left(\delta_{1}, \delta_{2}\right)$ be the maximal interval such that $d(\delta)>E(0)$ for $\delta \in\left(\delta_{2}, \delta_{2}\right)$. Then:

(i) All weak solutions of problem (1.1)-(1.3) belong to $W_{\delta}$ for $\delta \in\left(\delta_{1}, \delta_{2}\right)$ provided that $u_{0} \in W^{\prime}$.

(ii) All weak solutions of problem (1.1)-(1.3) belong to $V_{\delta}$ for $\delta \in\left(\delta_{1}, \delta_{2}\right)$ provided that $u_{0} \in V^{\prime}$.

3. Global existence of solutions. In this section we shall give some new existence theorems for global weak and strong solutions respectively for problem (1.1) -(1.3), which are different from those in [11. First we consider the existence of global weak solutions for problem (1.1)-(1.3) in the following theorem.

Theorem 3.1. Let $f(u)$ satisfy $\left(\mathrm{H}_{1}\right), u_{0} \in H, u_{1} \in L^{2}(\Omega)$. Assume that $E(0)<d$ and $u_{0} \in W^{\prime}$. Then problem (1.1)-(1.3) admits a global solution $u \in L^{\infty}(0, \infty ; H)$ with $u_{t} \in L^{\infty}\left(0, \infty ; L^{2}(\Omega)\right) \cap L^{2}\left(0, \infty ; H_{0}^{1}(\Omega)\right)$ and $u \in W$ for $0 \leq t<\infty$.

Proof. Let $\left\{w_{j}(x)\right\}_{j=1}^{\infty}$ be the eigenfunction system of problem $-\Delta w=\lambda w$ in $\Omega$, $\left.w\right|_{\partial \Omega}=0$. Construct the approximate solutions of problem (1.1)-(1.3):

$$
u_{m}(x, t)=\sum_{j=1}^{m} g_{j m}(t) w_{j}(x), \quad m=1,2, \ldots,
$$


satisfying

$$
\begin{aligned}
& \left(u_{m t t}, w_{s}\right)+\left(\nabla u_{m}, \nabla w_{s}\right)+\left(\Delta u_{m}, \Delta w_{s}\right)+\alpha\left(\nabla u_{m t}, \nabla w_{s}\right)=\left(f\left(u_{m}\right), w_{s}\right), \\
& s=1,2, \ldots, m \\
& u_{m}(x, 0)=\sum_{j=1}^{m} a_{j m} w_{j}(x) \rightarrow u_{0} \text { in } H \\
& u_{m t}(x, 0)=\sum_{j=1}^{m} b_{j m} w_{j}(x) \rightarrow u_{1} \text { in } L^{2}(\Omega) .
\end{aligned}
$$

Multiplying (3.2) by $g_{s m}^{\prime}(t)$ and summing for $s$ we get

$$
\frac{\mathrm{d}}{\mathrm{d} t} E_{m}(t)+\alpha\left\|\nabla u_{m t}\right\|^{2}=0
$$

and

$$
\frac{1}{2}\left\|u_{m t}\right\|^{2}+J\left(u_{m}\right)+\alpha \int_{0}^{t}\left\|\nabla u_{m \tau}\right\|^{2} \mathrm{~d} \tau=E_{m}(0), \quad 0 \leq t<\infty
$$

where

$$
E_{m}(t)=\frac{1}{2}\left\|u_{m t}\right\|^{2}+J\left(u_{m}\right) .
$$

From $E(0)<d, u_{0} \in W^{\prime}$ and (3.3), (3.4) it follows that $E_{m}(0)<d$ and $u_{m}(0) \in W^{\prime}$ for sufficiently large $m$. Then from (3.5) and the argument in the proof of Theorem 3.1 in [16] we can obtain that $u_{m}(t) \in W^{\prime}$ for $0 \leq t<\infty$ and sufficiently large $m$. Thus from (3.5) and

we get

$$
J\left(u_{m}\right) \geq \frac{p-1}{2(p+1)}\left\|u_{m}\right\|_{H}^{2}+\frac{1}{p+1} I\left(u_{m}\right) \geq \frac{p-1}{2(p+1)}\left\|u_{m}\right\|_{H}^{2}
$$

$$
\frac{1}{2}\left\|u_{m t}\right\|^{2}+\frac{p-1}{2(p+1)}\left\|u_{m}\right\|_{H}^{2}+\alpha \int_{0}^{t}\left\|\nabla u_{m \tau}\right\|^{2} \mathrm{~d} \tau<d, \quad 0 \leq t<\infty
$$

for sufficiently large $m$. Hence we have

$$
\begin{gathered}
\left\|u_{m}\right\|_{H}^{2} \leq \frac{2(p+1)}{p-1} d, \quad\left\|u_{m t}\right\|^{2} \leq 2 d, 0 \leq t<\infty \\
\int_{0}^{t}\left\|\nabla u_{m \tau}\right\|^{2} \mathrm{~d} \tau \leq \frac{d}{\alpha}, 0 \leq t<\infty \\
\left\|u_{m}\right\|_{q+1}^{2} \leq C_{*}^{2}\left\|u_{m}\right\|_{H}^{2} \leq C_{*}^{2} \frac{2(p+1)}{p-1} d, 0 \leq t<\infty \\
\left\|f\left(u_{m}\right)\right\|_{r+1}^{r+1} \leq \int_{\Omega}\left(A\left|u_{m}\right|^{q}\right)^{r} \mathrm{~d} x=A_{1}\left\|u_{m}\right\|_{q+1}^{q+1} \leq C_{*}^{q+1}\left(\frac{2(p+1)}{p-1} d\right)^{\frac{q+1}{2}}, \\
0 \leq t<\infty, r=\frac{q+1}{q} .
\end{gathered}
$$

Hence there exist a $u$ and a subsequence $\left\{u_{\nu}\right\}$ of $\left\{u_{m}\right\}$ such that as $\nu \rightarrow \infty$,

$u_{\nu} \rightarrow u$ in $L^{\infty}(0, \infty ; H)$ weakly star and a.e. in $Q=\Omega \times[0, \infty)$,

$u_{\nu} \rightarrow u$ in $L^{q+1}(\Omega)$ strongly for each $t>0$,

$u_{\nu t} \rightarrow u_{t}$ in $L^{\infty}\left(0, \infty ; L^{2}(\Omega)\right)$ weakly star,

$f\left(u_{\nu}\right) \rightarrow \chi$ in $L^{\infty}\left(0, \infty ; L^{r}(\Omega)\right)$ weakly star, 
$u_{\nu t} \rightarrow u_{t}$ in $L^{2}\left(0, \infty ; H_{0}^{1}(\Omega)\right)$ weakly.

By Lemma 1.3 in 12 we have $\chi=f(u)$.

Integrating (3.2) with respect to $t$ we get

$$
\begin{gathered}
\left(u_{m t}, w_{s}\right)+\int_{0}^{t}\left(\nabla u_{m}, \nabla w_{s}\right) d \tau+\int_{0}^{t}\left(\Delta u_{m}, \Delta w_{s}\right) d \tau+\alpha\left(\nabla u_{m}, \nabla w_{s}\right) \\
=\int_{0}^{t}\left(f\left(u_{m}\right), w_{s}\right) d \tau+\left(u_{m t}(0), w_{s}\right)+\alpha\left(\nabla u_{m}(0), \nabla w_{s}\right) .
\end{gathered}
$$

In (3.6) for fixed $s$ letting $m=\nu \rightarrow \infty$ we obtain

$$
\begin{aligned}
\left(u_{t}, v\right) & +\int_{0}^{t}(\nabla u, \nabla v) d \tau+\int_{0}^{t}(\Delta u, \Delta v) d \tau+\alpha(\nabla u, \nabla v) \\
= & \int_{0}^{t}(f(u), v) d \tau+\left(u_{1}, v\right)+\alpha\left(\nabla u_{0}, \nabla v\right), \forall v \in H .
\end{aligned}
$$

On the other hand, from (3.3) and (3.4) we have $u(x, 0)=u_{0}$ in $H$ and $u_{t}(x, 0)=u_{1}$ in $L^{2}(\Omega)$. Therefore $u(x, t)$ is a global weak solution of problem (1.1)-(1.3). Finally from Theorem 2.7 we get $u \in W$ for $0 \leq t<\infty$.

Next we try to give the existence theorem of global strong solutions for problem (1.1) (1.3). But here we would like to prove the main theorem in another way from Theorem 3.1. We shall give some lemmas first. Then we will show the key theorem (Theorem 3.5) as follows readily.

Let $f(u)$ satisfy $\left(\mathrm{H}_{2}\right), u_{0} \in H_{4}, u_{1} \in H,\left\{w_{j}(x)\right\}_{j=1}^{\infty}$ be the same as those in the proof of Theorem 3.1. Construct the approximate solutions $u_{m}$ by (3.1) satisfying

$$
\begin{aligned}
& \left(u_{m t t}-\Delta u_{m}+\Delta^{2} u_{m}-\alpha \Delta u_{m t}-f\left(u_{m}\right), w_{s}\right)=0, s=1,2, \ldots, m, \\
& u_{m}(x, 0)=\sum_{j=1}^{m} a_{j m} w_{j}(x) \rightarrow u_{0}(x) \text { in } H^{4}(\Omega), \\
& u_{m t}(x, 0)=\sum_{j=1}^{m} b_{j m} w_{j}(x) \rightarrow u_{1}(x) \text { in } H^{2}(\Omega) .
\end{aligned}
$$

First by the proof of Theorem 3.1 we can obtain the following lemma.

Lemma 3.2. Let $f(u)$ satisfy $\left(\mathrm{H}_{2}\right), u_{0} \in H_{4}, u_{1} \in H$. Assume that $E(0)<d$ and $u_{0} \in W^{\prime}$. Then for the approximate solutions $u_{m}$ defined by (3.1) and (3.8)-(3.10) we have

$$
\left\|u_{m}\right\|_{H}^{2} \leq \frac{2(p+1)}{p-1} d, \quad\left\|u_{m t}\right\|^{2} \leq 2 d, 0 \leq t<\infty .
$$

Lemma 3.3. Under the conditions of Lemma 3.2 we further have

$$
\left\|u_{m t}\right\|_{H}^{2}+\left\|u_{m}\right\|_{H_{4}}^{2} \leq C(T), 0 \leq t<\infty, \forall T>0 .
$$

Proof. Multiplying (3.8) by $\lambda_{s}^{2} g_{s m}^{\prime}(t)$ and summing for $s$ we get

$$
\frac{\mathrm{d}}{\mathrm{d} t}\left(\frac{1}{2}\left\|\Delta u_{m t}\right\|^{2}+\frac{1}{2}\left\|u_{m}\right\|_{H_{4}}^{2}\right)+\alpha\left\|\nabla^{3} u_{m t}\right\|^{2}=\left(f\left(u_{m}\right), \Delta^{2} u_{m t}\right),
$$




$$
\begin{aligned}
& \left(f\left(u_{m}\right), \Delta^{2} u_{m t}\right)=-\left(f^{\prime}\left(u_{m}\right) \nabla u_{m}, \nabla^{3} u_{m t}\right) \leq\left\|f^{\prime}\left(u_{m}\right)\right\|_{r}\left\|\nabla u_{m}\right\|_{s}\left\|\nabla^{3} u_{m t}\right\|, \\
& 1<r, s<\infty, \frac{1}{r}+\frac{1}{s}=\frac{1}{2} .
\end{aligned}
$$

(i) If $n \geq 7$, taking $s=\frac{2 n}{n-6}, r=\frac{n}{3}$, we get

$$
\begin{gathered}
\left\|\nabla u_{m}\right\|_{s} \leq C\left\|u_{m}\right\|_{H_{4}}, \\
\left\|f^{\prime}\left(u_{m}\right)\right\|_{r}^{r} \leq \int_{\Omega}\left(a\left|u_{m}\right|^{q_{1}}\right)^{\frac{n}{3}} \mathrm{~d} x=a^{\frac{n}{3}} \int_{\Omega}\left|u_{m}\right|^{q_{1} \cdot \frac{n}{3}} \mathrm{~d} x .
\end{gathered}
$$

From $\left(\mathrm{H}_{2}\right)$ we have $q_{1} \cdot \frac{n}{3} \leq \frac{2 n}{n-4}$. Hence from Lemma 3.2 we get $\left\|f^{\prime}\left(u_{m}\right)\right\|_{r} \leq C$ and

$$
\left(f\left(u_{m}\right), \Delta^{2} u_{m t}\right) \leq C\left\|u_{m}\right\|_{H_{4}}\left\|\nabla^{3} u_{m t}\right\| \leq \frac{C}{2 \alpha}\left\|u_{m}\right\|_{H_{4}}^{2}+\frac{\alpha}{2}\left\|\nabla^{3} u_{m t}\right\|^{2} .
$$

(ii) If $n=5,6$, take $s$ sufficiently large such that $q_{1} r \leq \frac{2 n}{n-4}$. Then again we get $\left\|f^{\prime}\left(u_{m}\right)\right\|_{r} \leq C$ and (3.14)

(iii) If $1 \leq n \leq 4$, take $s=r=4$. Then we again get (3.14).

Substituting (3.14) into (3.13) and then integrating the inequality with respect to $t$, by (3.3), (3.4) and the Gronwall inequality we can get (3.12).

LEMma 3.4. Under the conditions of Lemma 3.2 we further have

$$
\left\|u_{m t t}\right\| \leq C(T), \quad 0 \leq t<T, \forall T>0 .
$$

Proof. Multiplying (3.8) by $g_{s m}^{\prime \prime}(t)$ and summing for $s$ we get

$$
\begin{aligned}
\left\|u_{m t t}\right\|^{2} & =\left(\Delta u_{m}-\Delta^{2} u_{m}+\alpha \Delta u_{m t}+f\left(u_{m}\right), u_{m t t}\right) \\
& \leq\left(\left\|\Delta u_{m}\right\|+\left\|\Delta^{2} u_{m}\right\|+\alpha\left\|\Delta u_{m t}\right\|+\left\|f\left(u_{m}\right)\right\|\right)\left\|u_{m t t}\right\|, \quad 0 \leq t \leq T .
\end{aligned}
$$

From (ii) in $\left(\mathrm{H}_{2}\right)$ we can get

$$
|f(u)| \leq \frac{a}{q_{1}+1}|u|^{q_{1}+1} .
$$

So by (3.12) it is easy to see that $\left\|f\left(u_{m}\right)\right\| \leq C(T)$ for $0 \leq t \leq T$ and $\forall T>0$, which combining with (3.12) and Lemma 3.3 give (3.15).

From Lemma 3.2 Lemma 3.4 it is easy to get the following theorem.

Theorem 3.5. Let $f(u)$ satisfy $\left(\mathrm{H}_{2}\right), u_{0} \in H_{4}, u_{1} \in H$. Assume that $E(0)<d$, $I\left(u_{0}\right)>0$ or $u=0$, i.e. $u_{0} \in W^{\prime}$. Then problem (1.1)-1.3) admits a global strong solution $u \in L^{\infty}\left(0, T ; H_{4}\right) \cap L^{\infty}(0, \infty ; H)$ with $u_{t} \in L^{\infty}(0, T ; H) \cap L^{\infty}\left(0, \infty ; L^{2}(\Omega)\right)$, $u_{t t} \in L^{\infty}\left(0, T ; L^{2}(\Omega)\right), \forall T>0$ and $u \in W$ for $0 \leq t<\infty$.

4. Asymptotic behaviour of solutions. In this section we prove the asymptotic behaviour of global weak solutions for problem (1.1)-(1.3).

Lemma 4.1. Let $f(u)$ satisfy $\left(\mathrm{H}_{1}\right), u_{0} \in H, u_{1} \in L^{2}(\Omega), u_{m}$ be the approximate solutions given in the proof of Theorem 3.1, Then:

(i)

$$
I\left(u_{m}\right)=\left\|u_{m t}\right\|^{2}-\frac{\mathrm{d}}{\mathrm{d} t}\left(u_{m t}, u_{m}\right)-\frac{\alpha}{2} \frac{\mathrm{d}}{\mathrm{d} t}\left\|\nabla u_{m}\right\|^{2}, \forall m \text { and } 0 \leq t<\infty .
$$


(ii) Furthermore if $0<E(0)<d, u_{0} \in W^{\prime}$, then $u_{m} \in W_{\delta}$ for $\delta \in\left(\delta_{1}, \delta_{2}\right)$ and

$$
I\left(u_{m}\right) \geq\left(1-\delta_{1}\right)\left\|u_{m}\right\|_{H}^{2}
$$

for $0 \leq t<\infty$ and sufficiently large $m$, where $\left(\delta_{1}, \delta_{2}\right)$ is the maximal interval such that $d(\delta)>E(0)$ for $\delta \in\left(\delta_{1}, \delta_{2}\right)$.

Proof. $\quad$ (i) Multiplying (3.2) by $g_{s m}(t)$ and summing for $s$ yields (4.1).

(ii) First from (3.5) we get

$$
\frac{1}{2}\left\|u_{m t}\right\|^{2}+J\left(u_{m}\right) \leq E_{m}(0)<d(\delta), \delta_{1}<\delta<\delta_{2}, 0 \leq t<\infty
$$

for sufficiently large $m$. From (4.3) we can prove that $u_{m}(t) \in W_{\delta}$ for $\delta \in\left(\delta_{1}, \delta_{2}\right)$, $0 \leq t<\infty$ and sufficiently large $m$ by an argument similar to that in the proof of Theorem 3.1 in [16]. Thus we have $I_{\delta}\left(u_{m}\right) \geq 0$ for $\delta \in\left(\delta_{1}, \delta_{2}\right)$ and $I_{\delta_{1}}\left(u_{m}\right) \geq 0$. Hence

$$
\begin{aligned}
I\left(u_{m}\right) & =\left\|u_{m}\right\|_{H}^{2}-\int_{\Omega} u_{m} f\left(u_{m}\right) \mathrm{d} x=\left(1-\delta_{1}\right)\left\|u_{m}\right\|_{H}^{2}+I_{\delta_{1}}\left(u_{m}\right) \\
& \geq\left(1-\delta_{1}\right)\left\|u_{m}\right\|_{H}^{2} .
\end{aligned}
$$

Theorem 4.2. Let $f(u)$ satisfy $\left(\mathrm{H}_{1}\right), u_{0} \in H, u_{1} \in L^{2}(\Omega)$. Assume that $0<E(0)<d$ and $u_{0} \in W^{\prime}$. Then for the global weak solution $u$ given in Theorem 3.1,

$$
\left\|u_{t}\right\|^{2}+\|u\|_{H}^{2} \leq C e^{-\lambda t}, 0 \leq t<\infty
$$

for some positive constants $C$ and $\lambda$.

Proof. First we prove that for the approximate solutions $u_{m}$ given in the proof of Theorem 3.1

$$
0 \leq E_{m}(t) \leq C e^{-\lambda t}, 0 \leq t<\infty
$$

for some positive constants $C$ and $\lambda$ and sufficiently large $m$, where

$$
E_{m}(t)=\frac{1}{2}\left\|u_{m t}\right\|^{2}+\frac{1}{2}\left\|u_{m}\right\|_{H}^{2}-\int_{\Omega} F\left(u_{m}\right) \mathrm{d} x
$$

Note that from the proof of Theorem 3.1 we have

$$
\frac{\mathrm{d}}{\mathrm{d} t} E_{m}(t)+\alpha\left\|\nabla u_{m t}\right\|^{2}=0 .
$$

Multiplying (4.6) by $e^{\gamma t}(\gamma>0)$ we get

$$
\frac{\mathrm{d}}{\mathrm{d} t}\left(e^{\gamma t} E_{m}(t)\right)+\alpha e^{\gamma t}\left\|\nabla u_{m t}\right\|^{2}=\gamma e^{\gamma t} E_{m}(t)
$$

and

$$
e^{\gamma t} E_{m}(t)+\alpha \int_{0}^{t} e^{\gamma \tau}\left\|\nabla u_{m \tau}\right\|^{2} \mathrm{~d} \tau=E_{m}(0)+\gamma \int_{0}^{t} e^{\gamma \tau} E_{m}(\tau) \mathrm{d} \tau .
$$

From Lemma 4.1 we have $u_{m}(t) \in W$ for $0 \leq t<\infty$ and sufficiently large $m$, which gives

$$
E_{m}(t)=\frac{1}{2}\left\|u_{m t}\right\|^{2}+J\left(u_{m}\right) \geq \frac{1}{2}\left\|u_{m t}\right\|^{2}+\frac{p-1}{2(p+1)}\left\|u_{m}\right\|_{H}^{2}+\frac{1}{p+1} I\left(u_{m}\right) \geq 0,0 \leq t<\infty .
$$


From Lemma 4.1 we get

$$
\begin{aligned}
& \int_{0}^{t} e^{\gamma \tau} E_{m}(\tau) \mathrm{d} \tau \leq \frac{1}{2} \int_{0}^{t} e^{\gamma \tau}\left(\left\|u_{m \tau}\right\|^{2}+\left\|u_{m}\right\|_{H}^{2}\right) \mathrm{d} \tau \\
& \leq \frac{1}{2} \int_{0}^{t} e^{\gamma \tau}\left(\left\|u_{m \tau}\right\|^{2}+\frac{1}{1-\delta_{1}} I\left(u_{m}\right)\right) \mathrm{d} \tau \\
& =\frac{1}{2}\left(1+\frac{1}{1-\delta_{1}}\right) \int_{0}^{t} e^{\gamma \tau}\left\|u_{m \tau}\right\|^{2} \mathrm{~d} \tau \\
& -\frac{1}{2\left(1-\delta_{1}\right)} \int_{0}^{t} e^{\gamma \tau} \frac{\mathrm{d}}{\mathrm{d} \tau}\left(\left(u_{m \tau}, u_{m}\right)+\frac{\alpha}{2}\left\|\nabla u_{m}\right\|^{2}\right) \mathrm{d} \tau
\end{aligned}
$$

and

$$
\begin{aligned}
&-\int_{0}^{t} e^{\gamma \tau} \frac{\mathrm{d}}{\mathrm{d} \tau}\left(\left(u_{m \tau}, u_{m}\right)+\frac{\alpha}{2}\left\|\nabla u_{m}\right\|^{2}\right) \mathrm{d} \tau \\
&=\left(u_{m t}(0), u_{m}(0)\right)+\frac{\alpha}{2}\left\|\nabla u_{m}(0)\right\|^{2}-e^{\gamma t}\left(\left(u_{m t}, u_{m}\right)+\frac{\alpha}{2}\left\|\nabla u_{m}\right\|^{2}\right) \\
&+\gamma \int_{0}^{t} e^{\gamma \tau}\left(\left(u_{m \tau}, u_{m}\right)+\frac{\alpha}{2}\left\|\nabla u_{m}\right\|^{2}\right) \mathrm{d} \tau \\
& \leq \frac{1}{2}\left(\left\|u_{m t}(0)\right\|^{2}+\left\|u_{m}(0)\right\|^{2}+\alpha\left\|\nabla u_{m}(0)\right\|^{2}\right) \\
&+\frac{1}{2} e^{\gamma t}\left(\left\|u_{m t}\right\|^{2}+\left\|u_{m}\right\|^{2}+\alpha\left\|\nabla u_{m}\right\|^{2}\right) \\
&+\frac{\gamma}{2} \int_{0}^{t} e^{\gamma \tau}\left(\left\|u_{m \tau}\right\|^{2}+\left\|u_{m}\right\|^{2}+\alpha\left\|\nabla u_{m}\right\|^{2}\right) \mathrm{d} \tau
\end{aligned}
$$

From (4.8) we get

$$
E_{m}(t) \geq \frac{1}{2}\left\|u_{m t}\right\|^{2}+\frac{p-1}{2(p+1)}\left\|u_{m}\right\|_{H}^{2} .
$$

Hence there exists a $C>0$ such that

$$
\frac{1}{2}\left(\left\|u_{m t}\right\|^{2}+\left\|u_{m}\right\|^{2}+\alpha\left\|\nabla u_{m}\right\|^{2}\right) \leq C E_{m}(t), 0 \leq t<\infty .
$$

Thus from (4.7), (4.9), (4.10) and (4.11) it follows that there exist positive constants $C_{0}$ and $C_{1}$ such that

$$
\begin{aligned}
e^{\gamma t} E_{m}(t) & +\alpha \int_{0}^{t} e^{\gamma \tau}\left\|\nabla u_{m \tau}\right\|^{2} \mathrm{~d} \tau \\
\leq & C_{0} E_{m}(0)+\frac{\lambda_{0} \gamma}{2}\left(1+\frac{1}{1-\delta_{1}}\right) \int_{0}^{t} e^{\gamma \tau}\left\|\nabla u_{m \tau}\right\|^{2} \mathrm{~d} \tau+C_{1} \gamma e^{\gamma \tau} E_{m}(t) \\
& +C_{1} \gamma^{2} \int_{0}^{t} e^{\gamma \tau} E_{m}(\tau) \mathrm{d} \tau, \quad 0 \leq t<\infty
\end{aligned}
$$

where

$$
\lambda_{0}=\sup _{\substack{u \in H \\ u \neq 0}} \frac{\|u\|^{2}}{\|\nabla u\|^{2}} .
$$


Take $\gamma$ such that

$$
0<\gamma<\min \left\{\frac{1}{2 C_{1}}, \frac{2 \alpha}{\lambda_{0}\left(1+\frac{1}{1-\delta_{1}}\right)}\right\}
$$

Then (4.12) gives

$$
e^{\gamma t} E_{m}(t) \leq 2 C_{0} E_{m}(0)+2 C_{1} \gamma^{2} \int_{0}^{t} e^{\gamma \tau} E_{m}(\tau) \mathrm{d} \tau
$$

and

$$
e^{\gamma t} E_{m}(t) \leq 2 C_{0} E_{m}(0) e^{2 C_{1} \gamma^{2} t}<2 C_{0} d e^{2 C_{1} \gamma^{2} t}, 0 \leq t<\infty
$$

for sufficiently large $m$, which gives (4.5), where $C=2 C_{0} d, \lambda=\gamma\left(1-2 C_{1} \gamma\right)>0$.

From (4.11) we get that for sufficiently large $m$,

$$
\left\|u_{m t}\right\|^{2}+\left\|u_{m}\right\|_{H}^{2} \leq \frac{2(p+1)}{p-1} E_{m}(t), 0 \leq t<\infty .
$$

Let $\left\{u_{\nu}\right\}$ be the subsequence of $\left\{u_{m}\right\}$ given in the proof of Theorem 3.1. Then we have

$$
\begin{aligned}
\left\|u_{t}\right\|^{2}+\|u\|_{H}^{2} & \leq \liminf _{\nu \rightarrow \infty}\left(\left\|u_{\nu t}\right\|^{2}+\left\|u_{\nu}\right\|_{H}^{2}\right) \\
& \leq \liminf _{\nu \rightarrow \infty} \frac{2(p+1)}{p-1} E_{\nu}(t) \leq \frac{2(p+1)}{p-1} C e^{-\lambda t}, 0 \leq t<\infty,
\end{aligned}
$$

which gives (4.4).

Corollary 4.3. Under the conditions of Theorem 4.2.

$$
\|u\|_{r}^{2} \leq C e^{-\lambda t}, 0 \leq t<\infty
$$

for some positive constants $C$ and $\lambda$, where $1 \leq r \leq \frac{2 n}{n-4}$ for $n \geq 5 ; 1 \leq r<\infty$ for $n=4$; $1 \leq r \leq \infty$ for $n \leq 3$. In particular, if $n \leq 3$, then

$$
\|u\|_{\infty}^{2} \leq C e^{-\lambda t}, 0 \leq t<\infty
$$

Inequality (4.13) shows that the amplitude or oscillation of the wave described by problem (1.1) -(1.3) and the solutions of problem (1.1)-(1.3) decay to zero exponentially as time $t$ tends to infinity.

5. Examples. In this section we shall give some explicit examples of nonlinearities $f$ satisfying assumptions $(\mathrm{H} 1)$ and $(\mathrm{H} 2)$.

EXAMPLE 5.1. If

$$
f(u)=a u^{2 k+1}, \quad k=1,2, \ldots,
$$

where $a>0, p=q=2 k+1$ satisfies the conditions in (H1), then $f(u)$ satisfies (H1).

EXAMPLE 5.2. If

$$
f(u)=u^{q_{1}+1},
$$

where $p=q_{1}+1$ satisfies the conditions in (H2), then $f(u)$ satisfies (H2). 
Acknowledgement. This work was supported by the National Natural Science Foundation of China (11101102), Ph.D. Programs Foundation of Ministry of Education of China (20102304120022), the Support Plan for the Young College Academic Backbone of Heilongjiang Province (1252G020), the Natural Science Foundation of Heilongjiang Province (A201014), Foundational Science Foundation of Harbin Engineering University and Fundamental Research Funds for the Central Universities (HEUCF20131101).

\section{REFERENCES}

[1] D.D. Ang and A.P.N. Dinh, On the strongly damped wave equation $u_{t t}-\Delta u-\Delta u_{t}+f(u)=0$, SIAM J. Math. Anal. 19 (1988), 1409-1417. MR965260 (89j:35086)

[2] J. Arrieta, A.N. Carvalho, and J.K. Hale, A damped hyperbolic equation with critical exponent, Comm. Partial Differ. Eqs. 17 (1992), 841-866. MR.1177295 (93f:35145)

[3] V. Belleri and V. Pata, Attractors for semilinear strongly damped wave equation on $R^{3}$, Discrete Contin. Dynam. Systems 7 (2001), 719-735. MR.1849655 (2003f:35026)

[4] A.N. Carvalho and J.W. Cholewa, Local well posedness for strongly damped wave equations with critical nonlinearities, Bull. Austral. Math. Soc. 66 (2002), 443-463. MR.1939206 (2004b:35228)

[5] C. Chen and L. Ren, Weak solution for a fourth-order nonlinear wave equation, J. Southeast Univ. 21 (2005), 369-374. MR2174130

[6] J.A. Esquivel-Avila, Dynamics around the ground state of a nonlinear evolution equation, Nonlinear Anal. 63 (2005), 331-343.

[7] F. Gazzola and M. Squassina, Global solutions and finite time blow up for damped semilinear wave equations, Ann. Inst. H. Poincaré Anal. Non Linéaire 23 (2006), 185-207. MR2201151|(2007c:35118)

[8] S. Gerbi and B. Said-Houari, Exponential decay for solutions to semilinear damped wave equation, arXiv:0812.3637v3.

[9] H.A. Levine, P. Pucci, and J. Serrin, Some remarks on global nonexistence for nonautonomous abstract evolution equations, Contemp. Math. 208 (1997), 253-263. MR.1467010 (98j:34124)

[10] H.A. Levine and J. Serrin, Global nonexistence theorems for quasilinear evolution equations with dissipation, Arch. Rational Mech. Anal. 137 (1997), 341-361. MR1463799 (99b:34110)

[11] Q. Lin, Y. Wu, and S. Lai, On global solution of an initial boundary value problem for a class of damped nonlinear equations, Nonlinear Anal. 69 (2008), 4340-4351. MR2467236 (2010a:35170)

[12] J.L. Lions, Quelques méthodes de résolution des problèmes aux limites non linéaires, Dunod, Paris, 1969.

[13] Y. Liu, W. Feng, and D. Liu, Strongly damped nonlinear wave equation in arbitrary dimensions (1), Math. Appl. 8 (1995), 262-266. MR1359437 (96i:35088)

[14] Y. Liu and D. Liu, Initial boundary value problem of equation $u_{t t}-\alpha \Delta u_{t}-\Delta u=f(u)$, J. Huazhong Univ. Sci. Tech. 16 (1988), 169-173. MR1120240 (92f:35105)

[15] Y. Liu and P. Liu, On potential well and application to strongly damped nonlinear wave equations, Acta Math. Appl. Sin. 27 (2004), 710-722. MR2126318

[16] Y. Liu and R. Xu, Fourth order wave equations with nonlinear strain and source terms, J. Math. Anal. Appl. 331 (2007), 585-607. MR2306025 (2008c:35206)

[17] M. Ohta, Remarks on blowup of solutions for nonlinear evolution equations of second order, Adv. Math. Sci. Appl. 8 (1998), 901-910. MR.1657188 (99m:35167)

[18] K. Ono, On global existence, asymptotic stability and blowing up of solutions for some degenerate non-linear wave equations of Kirchhoff type with a strong dissipation, Math. Methods Appl. Sci. 20 (1997), 151-177. MR1430038 (97j:35102)

[19] V. Pata and M. Squassina, On the strongly damped wave equation, Comm. Math. Phys. 253 (2005), 511-533. MR2116726 (2005k:35291)

[20] V. Pata and S. Zelik, Smooth attractors for strongly damped wave equations, Nonlinearity 19 (2006), 1495-1506. MR2229785 (2007f:35257)

[21] P. Pucci and J. Serrin, Global nonexistence for abstract evolution equations with positive initial energy, J. Differential Equations 150 (1998), 203-214. MR.1660250 (2000a:34119)

[22] E. Vitillaro, Global existence theorems for a class of evolution equations with dissipation, Arch. Rational Mech. Anal. 149 (1999), 155-182. MR1719145 (2000k:35205) 
[23] G.F. Webb, Existence and asymptotic behavior for a strongly damped nonlinear wave equation, Canad. J. Math. 32 (1980), 631-643. MR586981 (81i:35116)

[24] R. Xu and Y. Liu, Asymptotic behavior of solutions for initial-boundary value problems for strongly damped nonlinear wave equations, Nonlinear Anal. 69 (2008), 2492-2495. MR2446346 (2009h:35292) 